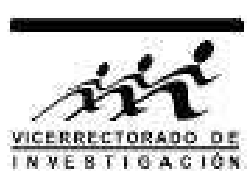

\title{
Caracterización climática de la circulación atmosférica en América del Sur
}

\author{
Miguel Saavedra ${ }^{* 1}$, Martín Calvo ${ }^{1}$ y César Jiménez ${ }^{1,2}$ \\ ${ }^{1}$ Laboratorio de Física de Eventos Naturales, Facultad de Ciencias Físicas, Universidad Nacional Mayor \\ de San Marcos, Lima, Perú \\ ${ }^{2}$ Dirección de Hidrografía y Navegación, Lima Perú
}

Recibido 15 noviembre 2011 - Aceptado 20 de diciembre 2011

\begin{abstract}
En el presente trabajo se realiza un estudio basado en los datos de Reanálisis para identificar los principales patrones de circulación atmosférica en sudamérica. Para el procesamiento de la información no se realiza ninguna distinción entre años El Niño y no El Niño, considerando solo promedios de los principales variables meteorológicas, para la identificación de patrones de la circulación atmosférica. Mediante el análisis de las gráficas de presión atmosférica y vientos, se identifica que la circulación superficial esta gobernada principalmente por la presencia de los anticiclones del Pacífico y Atlántico sur respectivamente, y a niveles altos el sistema denominado Alta de Bolivia, cuya influencia se observa a partir de $500 \mathrm{hPa}$. Por su parte, los patrones para la precipitación se destacan la Zona de Convergencia Intertropical, ZCIT, la Zona de Convergencia del Atlántico Sur, ZCAS, y los desiertos costeros que se ubican a lo largo de las costas de Perú y Chile. La ZCIT oscila cerca al Ecuador terrestre, con valores máximos de precipitación y con vientos zonales, producto de la convergencia de los sistemas de viento de los hemisferios norte y sur.
\end{abstract}

Palabras claves: Reanálisis, circulación atmosférica, anticiclón, viento, precipitación.

\section{Climatic caracterization of the atmospheric circulation in South America}

In the present paper, using the data obtained by Reanalysis we identify the main patterns of atmospheric circulation in South America. For the processing of information, we made not distinction between El Niño, and No El Niño years, considering only averages of the main meteorological variables for the patterns identification.

By analyzing the graphs of atmospheric pressure and winds, it was identified that the surface circulation is governed mainly by the presence of anticyclones in the Pacific and South Atlantic, and high levels of the system called Alta Bolivia, whose influence is seen from $500 \mathrm{hPa}$. On the other hand, the rainfall patterns highlight the Intertropical Convergence Zone, ITCZ, the Convergence Zone of the South Atlantic, SACZ, and the coastal deserts are located along the coasts of Peru and Chile. The ITCZ oscillates near the Equator, with maximum values of precipitation and zonal winds, resulting from the converge of the wind systems of the northern and southern hemispheres.

Keywords: Reanalysis, atmospheric circulation, anticyclone, wind, precipitation.

En la actualidad, las ciencias atmosféricas han cobrado gran importancia, debido a los cambios que viene experimentando el clima en nuestro planeta, lo que complementado con un gran desarrollo en recursos computacionales nos permite tener un nivel de comprensión cada vez mayor y mejor de los procesos físicos que ocurren en la atmósfera.

El sistema observacional a nivel mundial, viene siendo incrementado, principalmente en el Hemisferio Norte, HN, donde se encuentran la mayoría de países indus- trializados, ante la preocupación del cambio climático. En el Hemisferio Sur, HS, existe déficit en el sistema observacional, lo que ocasiona que en esta parte del planeta, los datos de observación in situ sean escasos, haciendo necesario que se recurra a los recursos que el desarrollo científico y tecnológico nos permite. Es así que se cuenta con información reconstruida mediante técnicas sofisticadas por el proyecto Reanalisis desarrollado por el National Centers for Environmental Prediction, NCEP, y el National Center for Atmospheric Research,

*miguel.saavedra.jded@hotmail.com 
NCAR, el mismo que nos permite disponer de una base de datos de buena calidad. De otro lado y gracias al desarrollo computacional, se dispone de herramientas de modelamiento numérico, las cuales nos permite cubrir grandes áreas con datos generados a partir de soluciones numéricas de las ecuaciones que gobiernan la dinámica de los parámetros físicos de interés.

En este contexto se presenta el presente estudio, con el objetivo de conocer con mayor precisión los patrones climáticos de la circulación atmosférica de América del Sur. Este conocimiento, permite entender desde el punto de vista de un modelo conceptual el comportamiento y posible pronóstico de las condiciones dinámicas de la atmosfera. De otro lado, el mismo, nos sirve como entrada para realizar técnicas de reducción de escala, downscaling, y analizar lo que sucede en la pequeña escala.

A mediano plazo, se espera que el presente trabajo, sirva como referente para el entendimiento de la dinámica de mesoescala a nivel de América del Sur, así como una motivación para continuar o complementar lo realizado, mediante el análisis de los demás parámetros climáticos, a diferentes niveles verticales.

Los datos de reanálisis, son obtenidos empleando técnicas de modelado numérico y asimilación de datos del sistema observacional, proporcionando información de calidad sobre el clima en toda la atmosfera global y regional, que nos permite estudiar procesos físicos y confirmar la validez de algunas hipótesis. No hay límite para las aplicaciones potenciales con estos datos, como por ejemplo, para estudios sobre cambio climático y sistemas de circulación atmosférica global o regional.

El modelado numérico se basa en la descripción de los fenómenos atmosféricos por medio de las leyes físicas de la hidrodinámica y termodinámica, considerando siete variables primarias como la presión, la temperatura, la densidad del aire, la humedad y las tres componentes de la velocidad del viento, y que se expresan mediante las ecuaciones [1,2] para la conservación de la masa del aire

$$
\frac{\partial \rho}{\partial t}=-\nabla \cdot \rho \boldsymbol{v}
$$

y la conservación de la cantidad de movimiento

$$
\frac{\partial \boldsymbol{v}}{\partial t}=-\boldsymbol{v} \cdot \nabla \boldsymbol{v}-\frac{1}{\rho} \nabla p-g \boldsymbol{k}-2 \boldsymbol{\Omega} \times \boldsymbol{v}+\boldsymbol{F}_{r},
$$

donde $\boldsymbol{v}$ es la velocidad, $t$ tiempo, $\rho$ la densidad, $p$ presión, $g$ aceleración de la gravedad y $\Omega$ es la velocidad angular de un punto sobre la superficie de la Tierra. El término $\partial \boldsymbol{v} / \partial t$, representa la aceleración local, $\boldsymbol{v} \cdot \nabla \boldsymbol{v}$ es la aceleración advectiva, $(1 / \rho) \nabla p$ es la fuerza debida al gradiente de presión, $g \boldsymbol{k}$ es el término gravitacional, $2 \boldsymbol{\Omega} \times \boldsymbol{v}$, es el término de Coriolis y $\boldsymbol{F}_{r}$ representa las fuerzas de fricción.
La ecuación de estado viene dado por

$$
p_{a}=\sum_{q} p_{q}=k_{\beta} T \sum_{q} N_{q}=N_{a} k_{\beta} T
$$

donde $p_{a}$ es la presión total, $N_{a}$ es la concentración del aire, $k_{\beta}$ es la constante de Boltzmann y $T$ es la temperatura.

La ecuación hidrostática se expresa como la variación de la presión con la altitud o en su forma diferencial como

$$
d p_{a}=-\rho_{a} g d z
$$

donde $p_{a}$ es la presión, $\rho_{a}$ la densidad, $g$ la gravedad y $z$ la altitud.

La ecuación de la energía termodinámica es expresado como

$$
\begin{gathered}
\frac{d \theta_{v}}{d t}+(\boldsymbol{v} \cdot \nabla) \theta_{v}=\frac{1}{\rho_{a}}\left(\nabla \cdot \rho_{a} \boldsymbol{K}_{h} \nabla\right) \theta_{v}+ \\
+\frac{\theta_{v}}{c_{p, d} T_{v}} \sum_{n=1}^{N_{e, h}} \frac{d Q_{n}}{d t}
\end{gathered}
$$

donde $\theta_{v}$ es la temperatura potencial virtual, $\boldsymbol{K}_{h}$ es el tensor de difusión turbulenta, $N_{e, h}$ es el número de fuentes y sumideros, $Q_{n}$ son las fuentes de energía diabática, $c_{p, d}$ es el calor específico del aire seco.

\section{Método}

Para la elaboración del presente trabajo se utilizaron datos de Reanálisis del NCAR-NCEP [3], para el periodo 1978 - 1998, que se encuentran disponibles con resolución temporal cada 6 horas y espacial de $2.5^{\circ}$ de latitud por $2.5^{\circ}$ de longitud, a diferentes niveles verticales de 1000, 925, 850, 700, 600, 500 y $300 \mathrm{hPa}$ para diferentes variables tales como la altura geopotencial, el flujo de calor latente, la radiación superficial de onda larga, la presión atmosférica, la humedad específica, la temperatura superficial, la precipitación, componentes $u$ y $v$ de la velocidad del viento, entre otras.

Para el procesamiento de la información y generación de gráficos, se emplearon programas y scripts, elaborados con el Grid Analysis and Display System, GrADS, y el Generic Mapping Tools, GMT.

\section{Área de estudio}

El ámbito geográfico en el que se realizó el estudio abarca el área comprendida entre los $120^{\circ} \mathrm{O}$ hasta los $20^{\circ} \mathrm{O}$ en longitud y $60^{\circ} \mathrm{S}$ hasta los $20^{\circ} \mathrm{N}$ en latitud. El área delimitada por estas coordenadas contienen gran parte de Centroamérica y la totalidad de Sudamérica, además de contener parte de Océano Pacífico suroriental y Atlántico suroccidental, vea la Fig.1. Además dentro del área seleccionada, se desarrollan la mayoría de 
procesos atmosféricos de mesoescala que tienen influencia directa sobre las condiciones atmosféricas en el territorio peruano.

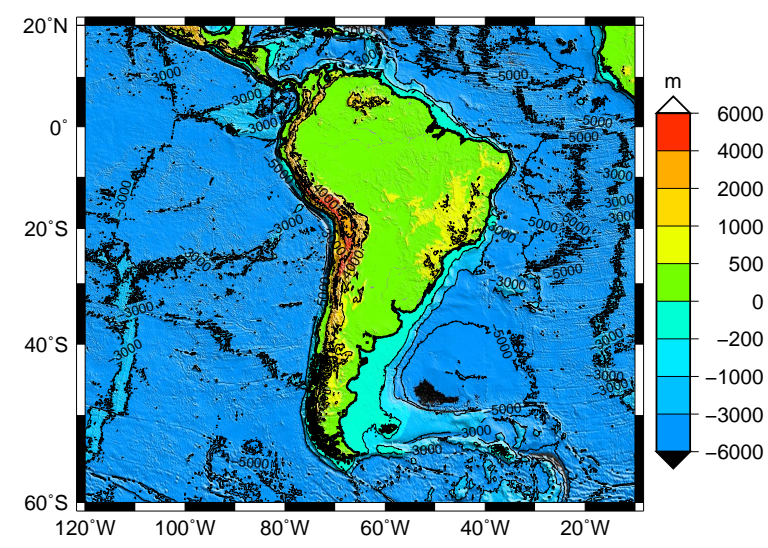

Figura 1: Topografía de Sudamérica, elaborada con los datos de ETOPO2.

\section{Descripción de los patrones climáticos de América del Sur}

La circulación atmosférica y el clima en el Pacífico Suroriental están controlados por la presencia de un sistema semipermanente de alta presión sobre el océano, cuyo núcleo se ubica frente a las costas del sur del Perú y norte de Chile. Este sistema conocido como el Anticiclón del Pacífico Sur, APS, produce condiciones muy estables en la tropósfera baja, temperaturas del mar relativamente bajas debido a los procesos de afloramiento, vientos predominantes del sur y una extensa cubierta de estratocúmulos, SC. Está cubierta de nubes, juega un papel importante en el balance superficial de radiación, al reflejar una parte importante de la radiación solar incidente, impidiendo el calentamiento de la superficie del mar y de la columna de aire que interactúa directamente con ella [4.

Superpuestas a este comportamiento estable de las condiciones medias, existen fluctuaciones de alta frecuencia, a escala diaria e intra-estacional, del espesor de la Capa Límite Marina, CLM, de la cobertura de SC, de la intensidad y la dirección del viento, de la temperatura superficial del mar, de la presión atmosférica y de otras variables. Estas fluctuaciones son particularmente marcadas en la franja costera y tienen un fuerte impacto en la meteorología regional [5].

Además, los fenómenos atmosféricos son fuertemente influenciados por la distribución de la topografía y la vegetación en la superficie del continente. Los diversos rasgos de las condiciones atmosféricas, el clima y la variabilidad climática sobre América del Sur, incluyen los rasgos de las latitudes tropicales, subtropicales y medias 6]. Otro fenómeno importante en esta región es El Niño y la Oscilación del Sur, que tiene su origen en el sistema acoplado océano-atmósfera en el Pacífico tropical, por lo que tiene una fuerte influencia sobre las áreas tropicales y subtropicales de América del Sur.

En la Figura 1, se muestra la topografía de América del Sur, con líneas de contorno en los 1000 y 4000 m para el continente y a los 200, 3000 y 4000 m de profundidad para el océano. En la figura mencionada, se puede observar que para la parte oceánica el ancho de la plataforma, a profundidades menores a $200 \mathrm{~m}$, sobrepasa fácilmente los $100 \mathrm{~km}$, en la parte circundante a Panamá. Se hace muy angosta entre Colombia y Ecuador, se ensancha en la parte norte de Perú, aproximadamente $100 \mathrm{~km}$, en la parte norte y central de Chile la plataforma se vuelve muy angosta y se ensancha nuevamente en el Sur.

En la parte continental, la Cordillera de los Andes se encuentra ubicada paralelamente a la costa Oeste del continente sudamericano desde el norte de Venezuela hasta la Patagonia en Argentina. Su parte más alta está localizada entre la línea ecuatorial y los $40^{\circ} \mathrm{S}$ de latitud. La placa continental tiene mayor extensión en su parte norte, disminuyendo considerablemente, a partir de los $20^{\circ} \mathrm{N}$, lo cual tiene efectos sobre la circulación atmosférica [7].

Otra característica importante de la topografía de América del Sur, es el Altiplano, ubicado en la zona central, entre los paralelos $15^{\circ} \mathrm{S}-21^{\circ} \mathrm{S}$, con una elevación promedio de $3700 \mathrm{~m}$. Esto, sumado a los efectos topográficos locales, constituye un factor determinante para el clima en esta región. Sobre la superficie, la presión atmosférica es aproximadamente $620 \mathrm{hPa}$, a $4000 \mathrm{~m}$, que contiene una densidad de aire $35 \%$ inferior al valor medido a nivel del mar y tiene valores reducidos de humedad atmosférica. La temperatura en la superficie es relativamente baja y su ciclo diario muestra una gran variabilidad, como resultado del fuerte calentamiento diurno y la considerable pérdida radiativa nocturna 8].

La Cordillera de los Andes representa un obstáculo formidable para la circulación atmosférica en la tropósfera, actuando como una barrera climática y obstaculizando el paso de la humedad, permitiendo la existencia de condiciones secas al oeste y húmedas al este en los subtrópicos, los patrones se invierten en las latitudes medias. También acoge interacciones tropicalesextratropicales, especialmente a lo largo del lado este.

El Escudo Brasileño tiende a bloquear la circulación atmosférica de los niveles bajos sobre los subtrópicos en América del Sur. Otro aspecto importante es la extensa área de continente en las bajas latitudes $10^{\circ} \mathrm{N}-20^{\circ} \mathrm{S}$, que condiciona el desarrollo de intensa actividad convectiva que da origen a la selva tropical más grande del mundo, en la cuenca del Amazonas 8].

En la zona ecuatorial, debido a la constante insola- 
ción, se produce una fuerte evaporación diaria que determina la formación de las precipitaciones convectivas. La orientación de las cadenas montañosas contribuye a la entrada al continente de masas de aire polar y tropical provenientes del Océano Atlántico, pero actúa como una barrera para las masas de aire tropical provenientes del Océano Pacífico que descargan su humedad en las laderas occidentales de las cadenas montañosas a modo de precipitaciones orográficas y continúan como vientos secos; esta es la causa de la aridez en la Patagonia.

En las costas orientales de América del Sur, debido a su relieve menos accidentado las masas de aire húmedas no presentan dificultades de penetración por lo cual las precipitaciones son mayores. Otro factor importante para la comprensión de los tipos climáticos son las corrientes marinas cálidas y frías responsables de mayores o menores índices de humedad y de temperatura de las masas de aire.

Los vientos húmedos del Pacífico al pasar por la corriente fria de Humboldt, sufren una condensación por enfriamiento y precipitan en el océano determinando desiertos costeros, a lo largo de las costas de Perú y Chile. Las corrientes cálidas contribuyen a aumentar el índice de precipitaciones y la temperatura de las zonas costeras, como la corriente de Brasil y de las ecuatoriales 8 .

Similarmente anomalías de TSM en el Océano Atlántico tienen un profundo impacto sobre las condiciones atmosféricas y clima a lo largo de la costa este del continente Sudamericano. Wyrtki 9] muestra que el núcleo de los alisios del sureste varían interanualmente en área e intensidad, siendo debilitado durante la fase cálida de los eventos ENSO.

\section{Caracterización de la circulación atmos- férica en América del Sur, mediante da- tos de reanálisis}

En esta sección, se presenta los resultados del procesamiento de la información obtenida mediante datos de reanálisis, poniendo énfasis en el ciclo anual y campos promedios estacionales y mensuales de algunas variables meteorológicas. La circulación atmosférica de vientos, presión, etc., ha sido caracterizada utilizando los datos de Reanálisis de NCEP-NCAR 3], para el periodo comprendido entre los años 1978-1998, utilizando campos promedio, sin realizar ninguna distinción entre los años de El Niño y de no El Niño.

El clima regional está definido por las propiedades relevantes de los valores medios de los campos, que están forzados por las condiciones de frontera de la atmósfera, de la distribución de continente-océano, de la topografía continental y las variaciones de la radiación solar sobre la superficie, en el espacio y tiempo [8].
En la Fig. 2, se presenta la precipitación media anual y la velocidad del viento promediadas entre los niveles de $1000 \mathrm{hPa}$ hasta los $850 \mathrm{hPa}$. Los colores muestran la magnitud de la precipitación expresada en $\mathrm{mm}$ /día y los vectores muestran el valor de la magnitud y dirección de la velocidad de viento.

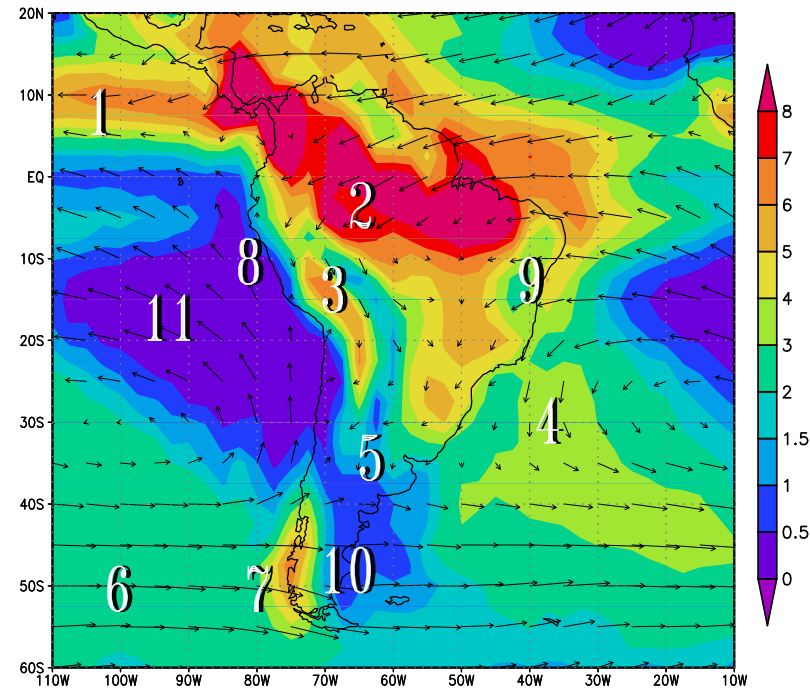

Figura 2: Promedios anuales de precipitación y velocidad del viento desde $1000 \mathrm{hPa}$ hasta $850 \mathrm{hPa}$. Los números indican las principales características de la precipitación [5].

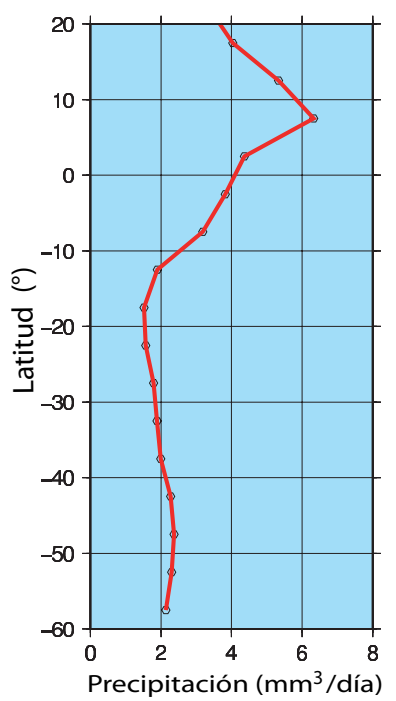

Figura 3: Variación de la precipitación con la latitud, promediada entre $101^{\circ}-10^{\circ} \mathrm{O}$ de longitud.

La Fig. 3 muestra la variación de la precipitación con la latitud, que ha sido obtenida al promediar los valores de precipitación entre los $110^{\circ} \mathrm{O}-10^{\circ} \mathrm{O}$ de longitud. Se observa que la ubicación de la máxima precipitación 
se encuentra aproximadamente a los $8^{\circ} \mathrm{N}$, y que la zona de convergencia de vientos llamada la Zona de Convergencia Intertropical, ZCIT, casi coincide con la región de máxima precipitación. Esta figura fue obtenida mediante la integración de la precipitación a lo largo de las franjas latitudinales, desde los $110^{\circ} \mathrm{O}$ hasta los $10^{\circ} \mathrm{O}$. En la Fig. 2, se enumera las principales características que tiene la precipitación en esta parte del planeta. En la Tabla 1, se resumen las principales características de la precipitación, obtenida de los promedios de los parámetros meteorológicos, basado en el trabajo de René Garreaud [8].

Los patrones que resaltan en la figura 2, son la ZCIT indicada con el número uno, que es la franja ubicada cerca a la línea ecuatorial, donde se presenta un alto nivel de precipitación, debido a que la humedad transportada desde el hemisferio norte y sur convergen en dicha zona.
Otros aspectos resaltantes que se pueden observar en la Fig. 2, son los desiertos costero y oceánico que se ubican a lo largo de Perú y Chile, en color azul, anotado con los números 8 y 11, respectivamente, donde los valores de la precipitación son prácticamente nulos, patrón que se extiende hacia una parte de la Patagonia. En estas áreas, debido a las bajas temperaturas del agua de mar, se generan condiciones de inversión térmica, que impiden el ascenso de masas de aire, produciendo condiciones desfavorables para la precipitación. Además, en esa misma figura se puede observar la convección continental, en la Fig. 2, con color rojo, indicado con el número 2 , ubicado en la selva tropical, donde se dan los máximos valores de precipitación, debido a que el aire húmedo y cálido del Océano Atlantico es transportado por los vientos alisios, con condiciones favorables para la precipitación. En la Tabla 1, se mencionan los demás patrones meteorológicos resaltados en la Fig. 2.

\begin{tabular}{ll|ll}
\hline & Precipitación (Figura 2) & & Circulación (Figura 4 y 5) \\
\hline 1 & ZCIT(ITCZ) & 1 & ZCIT(ITCZ) \\
2 & Convección Continental & 2 & Vientos Alisios \\
3 & Convección Altiplóanica & 3 & Alta Subtropical \\
4 & ZCAS (SACZ) & 4 & Vientos del Oeste \\
5 & Convección Pampas & 5 & "Low level jet" \\
6 & Mid latitude storm track & 6 & ZCAS (SACZ) \\
7 & Precipitación orográfica & 7 & Alta de Bolivia \\
8 & Desierto costero & 8 & Baja de NE Brasil \\
9 & Semiárida NE Brasil & 9 & Vientos tropicales del este \\
10 & Zona seca de Patagonia & 10 & Vientos del oeste \\
11 & Desierto oceánico & 11 & Jet stream \\
\hline
\end{tabular}

Tabla 1: Principales características de la precipitación y la circulación en Sudamérica, basada en el trabajo de René Garreaud [5].

En la Figura 4, se representan las principales características de la circulación atmosférica sobre Sudamérica, enumeradas en la tabla 1 en la columna de la derecha. Nuevamente resalta la ZCIT, donde convergen los sistemas de vientos de los hemisferios norte y sur, produciendo una franja de vientos con componentes netamente zonales. Otra característica notable de la circulación es el anticiclón del Pacífico y del Atlántico sur, que vienen a ser los centros de alta presión que controlan el sistema de vientos alisios en el Pacífico y en el Atlántico de esta región.

En la Figura 5, se representan las principales características de la circulación atmosférica en los niveles altos sobre Sudamérica, $300 \mathrm{hPa}$, donde se observa la Alta de Bolivia y la baja del NE de Brasil, marcados con los números 7 y 8 , respectivamente.

En las Figuras 6 y 7 se ha representado las carac- terísticas de la circulación superficial y la precipitación en las estaciones de verano e invierno. Como se puede observar en dichas figuras, el máximo de precipitación ocurre sobre el Pacífico tropical, a lo largo de la banda de aproximadamente $6^{\circ} \mathrm{N}$ de latitud, en el verano, y $10^{\circ} \mathrm{N}$ de latitud en el invierno, que coincide con el cinturón de baja presión y la convergencia de los vientos en la superficie, ZCIT, que viene a ser el rasgo de mayor importancia de la circulación global. Su variación anual, alrededor del Ecuador, está relacionada con la distribución continente-océano, la orientación de la línea de costa y el efecto de la Cordillera de los Andes.

La precipitación decrece levemente a ambos lados de la ITCZ, en parte debido al decrecimiento de la evaporación superficial, pero produce una alta precipitación continental sobre los Andes Ecuatoriales. Sobre la cuenca Oeste del Amazonas y cerca a la desembocadu- 
ra del río Amazonas, la precipitación normalmente es ocasionada por convección de la humedad que es la más energética para ascender en forma boyante desde la superficie hasta la tropopausa 8]. Además, la distribución de velocidades del viento sobre la costa peruana permite que la humedad atmosférica sea desplazada hacia el

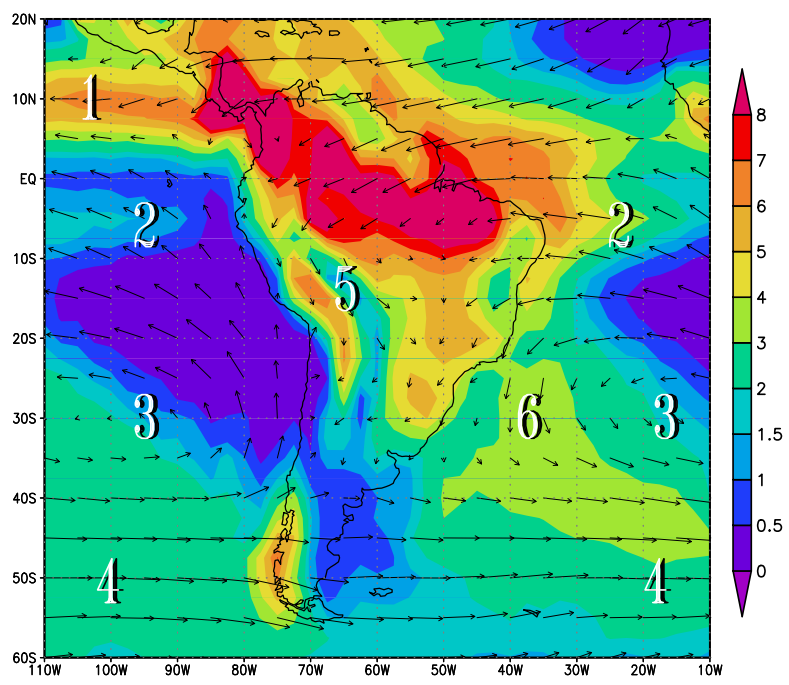

Figura 4: Promedios anuales de precipitación y velocidad del viento, promedios de $1000 \mathrm{hPa}$ a $850 \mathrm{hPa}$. Los números indican las características de la circulación, ver tabla 1.

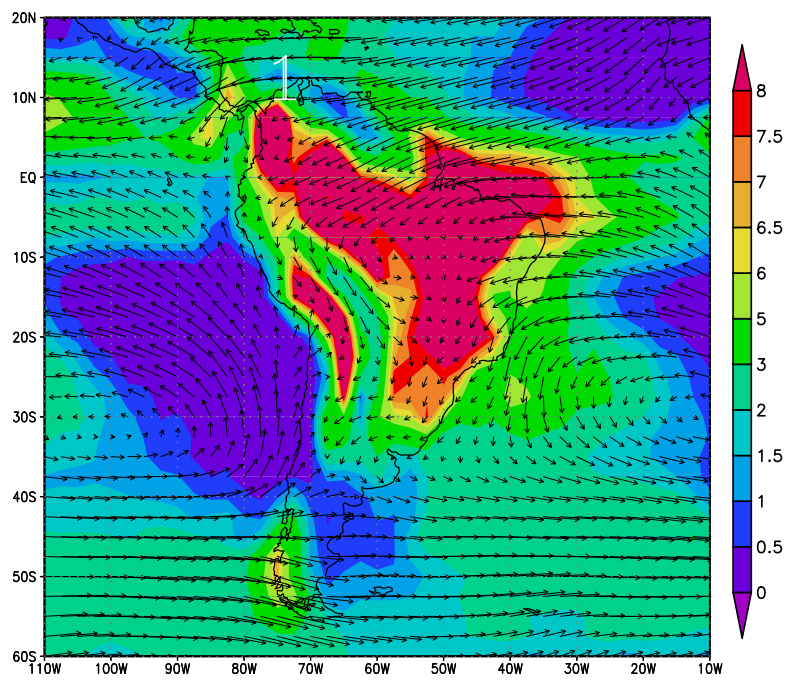

Figura 6: Precipitación en los meses de verano.

El desierto costero, en promedio se ubica entre los $8^{\circ} \mathrm{S}$ y los $30^{\circ} \mathrm{S}$, su extensión varía de estación en estación, ver Fig.4. Este desierto es considerado como el desierto costero más extenso, en longitud y seco de todo el mundo 10 .
Ecuador, originando la formación del desierto costero y oceánico a lo largo de las costas de Perú y Chile. Haciendo una digresión, en analogía a los desiertos costeros, se denomina desierto oceánico a las áreas oceánicas donde la precipitación es escasa, menor a $250 \mathrm{~mm}$ al año.

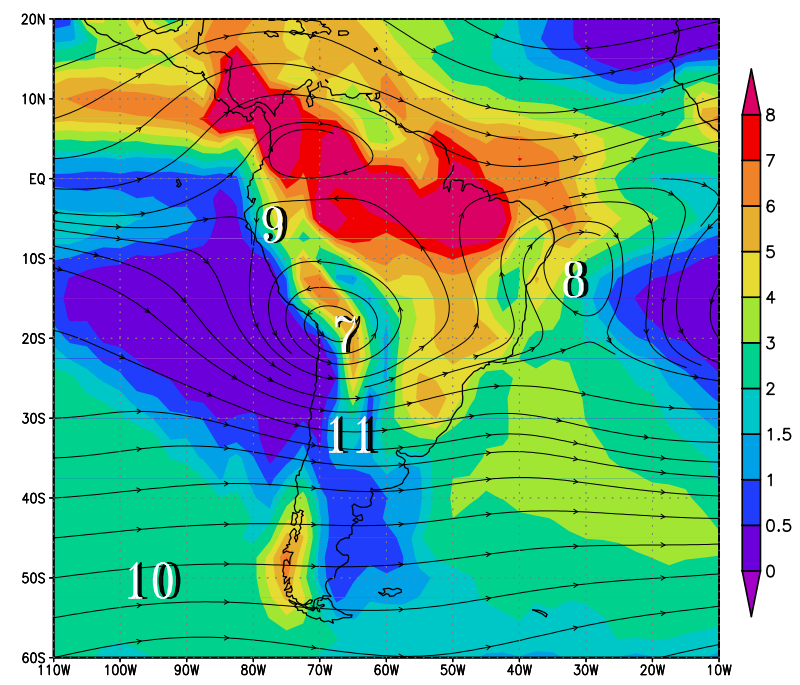

Figura 5: Precipitación y líneas de corriente para $300 \mathrm{hPa}$, promedio climatológico del mes de enero. Los números indican las características de la circulación, ver tabla 1.

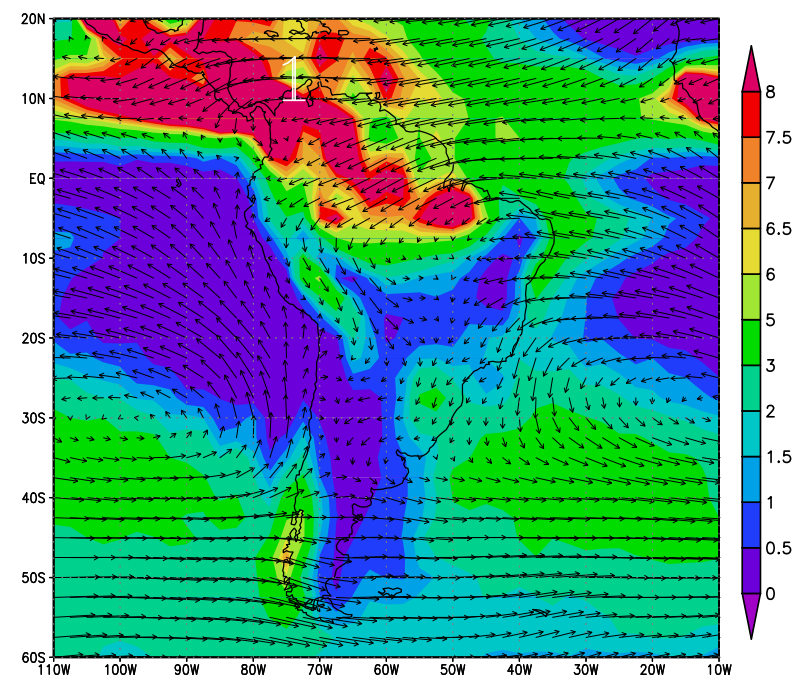

Figura 7: Precipitación en los meses de invierno.

\section{Conclusiones}

Los datos provenientes de Reanálisis, a pesar de tener una resolución espacial gruesa, sirven para estudiar y comprender muchas de las características de la cir- 
culación atmosférica alrededor del globo terrestre, y en particular para la región de Sudamérica.

A partir de los gráficos, obtenidos con los datos de Reanálisis se realizó la caracterización climática de la circulación atmosférica en Sudamérica, para identificar los principales patrones de circulación.

Las principales características de la precipitación en esta región son la Zona de Convergencia Intertropical, ZCIT, la Zona de Convergencia del Atlántico Sur, ZCAS, y los desiertos costeros que se ubican a lo largo de las costas de Perú y Chile. La ZCIT oscila cerca al Ecuador terrestre, con valores máximos de precipitación.

Mediante el análisis de las gráficas de las líneas de corriente de vientos, se identifica que la circulación superficial está gobernada por los anticiclones del Pacífico y del Atlántico sur, respectivamente; y a niveles altos el sistema denominado Alta de Bolivia ejerce una influencia que se observa a partir de $500 \mathrm{hPa}$. La ZCIT se caracteriza por contener vientos zonales, producto de la convergencia de los sistemas de viento de los hemisferios norte y sur.

\section{Agradecimientos}

Los autores agradecen al Instituto del Mar del Perú, donde fue realizado la mayor parte de este trabajo.

\section{Referencias}

[1] J. Holton; An Introduction to Dynamic Meteorology, fourth edition, Elsevier and Academic Press, Amsterdam (2004).

[2] M. Jacobson; Fundamentals of Atmospheric Modeling, second edition, Cambridge University Press, Cambridge (2005)

[3] E. Kalnay, M. Kanamitsu, R. Kistler, W. Collins, D. Deaven, L. Gandin, M. Iredell, S. Saha, G. White, J. Woollen, Y. Zhu, A. Leetmaa, R. Reynolds, M. Chelliah, W. Ebisuzaki, W.Higgins, J. Janowiak, K. C. Mo, C. Ropelewski y J. Wang; (NCEP Climate Prediction Center) Roy Jenne, Dennis Joseph, 1996; The NCEP/NCAR 40 Year Reanalysis Project, Bull. Amer. Meteor. Soc. 77, 437 (1996).

[4] R. Garreaud y R. Muñoz; Diurnal cycles of the circulation and low-cloud cover the subtropical southeastern Pacific; J. of Climate, 17, 699 (2004).

[5] R. Garreaud, J. Rutlland y H. Fuenzalida; Coastal lows along the subtropical west coast of South Ame- rica: Mean structure and evolution; Mon. Wea. Rev, 130, 75 (2002).

[6] P. Aceituno; Elementos del clima del Altiplano; Rev. UPGH. 44, 37 (1996).

[7] T, Strub, Jorge Mesias, Vivian Montecino, Jose Rutllant y Sergio Salinas; Coastal Ocean Circulation Off Western South America, The Sea, Vol.11, edited by Allan R. Robinson y Kennett H Brink; John Wiley \& Sons Inc., New York (1998).

[8] R. Garreaud y Patricio Aceituno; Atmospheric Circulation over South America: Mean features and variability, Capítulo 2, The Geography of South America; T. Vlemen, K. Young y A. Omme, Oxford University Press, Oxford (2001).

[9] K. Wyrtki; El Niño-the dynamic response of the equatorial Pacific Ocean to atmospheric forcing, J. Phys. Oceanogr. 5, 572 (1975).

[10] Magda Lucimar de Abreóu; Dynamics of the South American Coastal Desert. J. Atmosph. Sci. 50, 2952 (1993). 